\title{
THE BINDING OF ELECTRONS TO HEAVY IONS
}

\author{
E. VAN MEIJGAARD and Th.W. RUIJGROK \\ Instituut voor Theoretische Fysica, Rijksuniversiteit, Utrecht, The Netherlands*
}

Received 2 January 1985

Using a previously described relativistic quantum theory for two particles we calculate the spectrum of bound states of an electron in Coulomb interaction with a heavy ion of charge $Z$.

A spontaneous breakdown of the vacuum does not occur. We do find, however, a critical value of $Z$ beyond which electron-positron pairs will be produced. For this reaction to take place it is not necessary to consider a smeared out nuclear charge.

\section{Introduction}

The fine structure formula of Sommerfeld ${ }^{1}$ )

$$
E_{n k}=m c^{2}\left[1+\left\{\frac{Z \alpha}{n-k+\sqrt{k^{2}-Z^{2} \alpha^{2}}}\right\}^{2}\right]^{-1 / 2}
$$

with $n=1,2, \ldots$ and $k=1,2, \ldots, n$, predicts a breakdown of the spectrum if $Z$ becomes equal to the critical value $Z_{\mathrm{cr}}=\alpha^{-1}=137$.

The Dirac equation for an electron in the field of a nuclear point charge $Z$ miraculously gives exactly the same formula (S) for the spectrum, but with the integer $k$ taken as $j+\frac{1}{2}$. All energies lie between zero and $m c^{2}$ and the Dirac equation has no acceptable bound state solutions with negative energy.

Now, in the usual explanation ${ }^{2}$ ) of electron-positron pair production in heavy ion collisions, it is essential that with increasing $Z$ there exist single electron bound states with energies equal to or even less than $-m c^{2}$. This condition is met by assuming, not unrealistically, that the nuclear charge is spread over a finite region. In this note, however, we wish to show that, even for point charges, binding energies up to $2 m c^{2}$ can be obtained in a relativistic twoparticle theory proposed some time $\mathrm{ago}^{3,4}$ ).

* Mailing address: Princetonplein 5, Utrecht, The Netherlands. 
Also for the calculation of the fine structure of atomic spectra it is important to have a reliable and practical theory, which takes into account the existence of positrons. Brown and Ravenhall ${ }^{5}$ ) were the first to point out that beyond lowest order perturbation the existing theoretical method failed. A dissolution into the negative energy continuum takes place. These authors, and later $\mathrm{J}$. Sucher ${ }^{6}$ ), have given remedies for this failure. The theory used in this article in principle provides another solution to this problem, but will not be elaborated here.

\section{Binding energies}

In ref. 4 it was shown in great detail how a relativistic quantum theory for two and more particles could be constructed, which does not suffer from the Brown-Ravenhall disease. A number of examples was discussed, among which a relativistic version of the Lee model. This was done in order to show that also particle production could be incorporated.

In the present note we want to restrict ourselves to the Coulomb problem of bound states of two particles with masses $m$ and $M$, carrying charges $-e$ and $+Z e$ respectively. In $I$ the eigenvalue problem for $Z=1$ was derived and solved using perturbation theory. Now we want to consider the same problem for large values of $Z$ and in particular we wish to ascertain how strong the binding can be made by increasing the nuclear charge.

For this purpose we recall that, when recoil effects are omitted, the binding energy is given by $E_{\mathrm{b}}=m c^{2}(1+\cos \varepsilon)$, where $\varepsilon$ is to be solved from the eigenvalue equation ${ }^{7}$ )

$$
\frac{1}{\pi} \int_{0}^{\varepsilon} Q_{l}\left[\frac{1-\cos v \cos u}{\sin v \sin u}\right] \psi_{l}(u) \mathrm{d} u=\lambda_{l} \psi_{l}(v)
$$

together with

$$
\lambda_{l}=\frac{\sin \varepsilon}{Z \alpha} .
$$

$Q_{l}$ is a Legendre function of the second kind and $l$ is the quantum number for angular momentum. By expanding $\psi_{l}(u)$ in Gegenbauer functions

$$
\psi_{l}(u)=\sum_{k=0}^{\infty} \frac{c_{k}^{l+1}(u)}{\sqrt{k+l+1}} Y_{k}^{l},
$$


eq. (1) can also be written in matrix form:

$$
\sum_{k^{\prime}=0}^{\infty} M_{k k^{\prime}}^{l}(\varepsilon) Y_{k^{\prime}}^{\prime}=\lambda_{l} Y_{k}^{l}
$$

where

$$
M_{k k^{\prime}}^{l}(\varepsilon)=\frac{1}{\sqrt{(k+l+1)\left(k^{\prime}+l+1\right)}} \int_{0}^{\varepsilon} c_{k}^{l+1}(u) c_{k^{\prime}}^{l+1}(u) \mathrm{d} u
$$

can be calculated using certain relations for Gegenbauer polynomials ${ }^{8}$ ). We have solved eqs. (2) and (4) by truncating the dimension $N$ of the matrix $M^{l}$ at the value $N=100$. After choosing the value of $\varepsilon$ between 0 and $\pi$ the eigenvalues $\lambda_{l}(n)(n=1,2, \ldots, N)$ were calculated numerically. Fq. (2) then provided the corresponding value of $Z \alpha$. For $\varepsilon$ not too close to zero this method was stable under a change in $N$. For $\varepsilon \ll 1$ eq. (1) was transformed by writing $\lambda_{l}=\varepsilon \mu_{l}, v=\varepsilon x$ and $u=\varepsilon y$. In this way we got the eigenvalue problem for $\mu_{l}$ :

$$
\frac{1}{\pi} \int_{0}^{l} Q_{l}\left[\frac{1}{2}\left(\frac{x}{y}+\frac{y}{x}\right)\right] \psi_{l}(y) \mathrm{d} y=\mu_{l} \psi_{l}(x)
$$

This was solved numerically, giving for each $l$ a series of eigenvalues $\mu_{l}(n)$, $n=1,2, \ldots$ If we plot the left- and the right-hand side of eq. (2) as functions of $\varepsilon$ we obtain for $l=0$ the curves of fig. 1. The slope in $\varepsilon=0$ of $(\sin \varepsilon) / Z \alpha$ is $1 / Z \alpha$, whereas the slopes of the other curves are given by $\mu_{l}(n)$. For increasing $Z \alpha$ the amplitude of $(\sin \varepsilon) / Z \alpha$ decreases and each time $Z \alpha$ passes a critical value given by $Z_{\mathrm{cr}} \alpha-1 / \mu_{l}(n)$ a solution with $\varepsilon=0$ ceases to exist. This means that for each level the binding energy increases with the nuclear charge, until the value $2 m c^{2}$ is reached. The first time this occurs is at $Z_{\mathrm{cr}} \alpha=2$, i.e. for $Z_{\mathrm{cr}}=274$. For $l=0,1$ and 2 the results of the calculations are shown in fig. 2 . In the same figure we have plotted the binding energy according to the Sommerfeld formula (eq. (S)). For the Klein-Gordon equation the same fine structure formula eq. (S) holds ${ }^{9}$ ), but now with $k=l+\frac{1}{2}$ half-integer. As in the Dirac-theory there are no acceptable solutions with a binding energy larger than $m c^{2}$.

There is a large difference with our results, but this can only add to the lack of confidence one should have in the Klein-Gordon equation. Already in 1926 


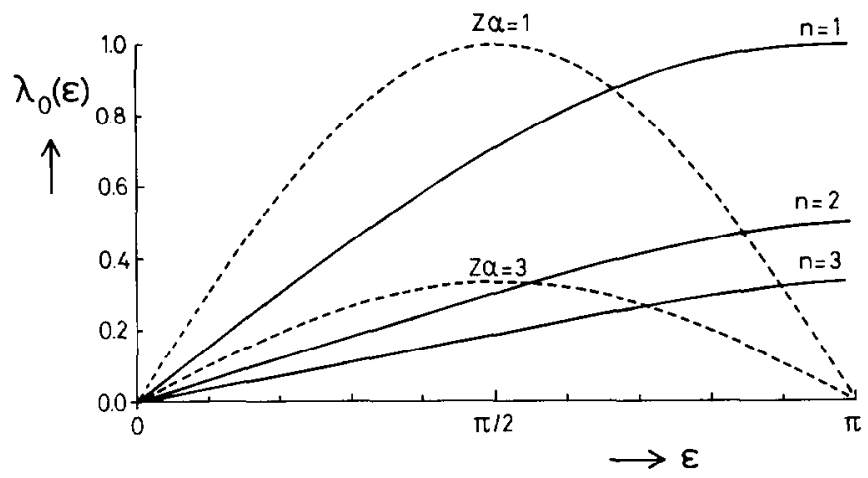

Fig. 1. Dashed lines $(\sin \varepsilon) / Z \alpha$ for $Z \alpha=1$ and $Z \alpha=3$. Full curves: Three eigenvalues $\lambda_{l}(\varepsilon)$ for $l=0$.

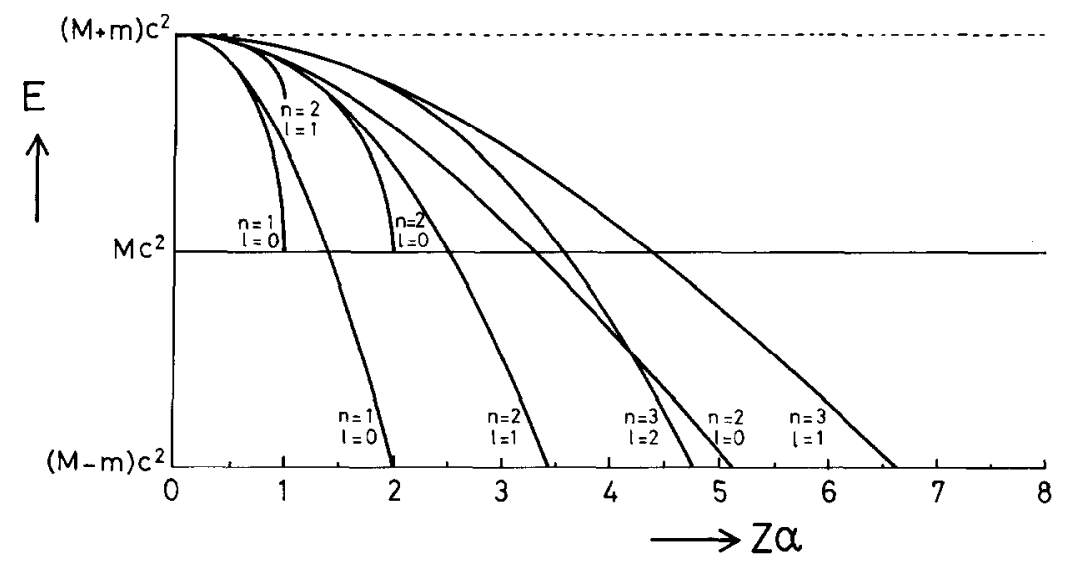

Fig. 2. Energy of different bound states as a function of $Z \alpha$.

Pauli observed ${ }^{10}$ ) that it is not self-adjoint and the eigenfunctions are therefore not orthogonal. In ref. 4 it is shown that this objection cannot be raised against the theory used for the present calculations.

Concluding we should like to make the following remarks.

1) The correct theory for the description of pair production in the neighbourhood of a supercritical point charge of course is Q.E.D. It has never been shown, however, and for a point charge it is certainly not true, that the Dirac equation gives a fair approximation to the binding energy.

2) A quasi-potential equation, like the one presented in this article, may come closer to reality, since it is free of internal inconsistencies. 


\section{References}

1) A. Sommerfeld, Ann. d. Phys. 51 (1916) 1.

2) J. Reinhardt, B. Müller and W. Greiner, Phys. Rev. A 24 (1981) 103 and further literature quoted there.

3) Th.W. Ruijgrok, Acta Physica Polonica B7 (1976) 43. This paper will be referred to as I

4) Th.W. Ruijgrok, Relativistic Quantum Mechanics, in: Quantum Theory of Particles and Fields, B. Jancewicz and J. Lukierski, eds. (World Scientific, Singapore, 1983), p. 117

5) G.E. Brown and D.G. Ravenhall, Proc. Roy. Soc. A208 (1951) 552.

6) J. Sucher, Phys. Rev. A 22 (1980) 348.

7) See I eqs. (54) and (55). $\varepsilon$ is changed into $\pi-\varepsilon$.

8) W. Magnus, F. Oberhettinger and R.P. Soni, Formulas and Theorems for the Special Functions of Mathematical Physics, 3rd ed. (Springer, Berlin, 1966), \$5.3.2.

9) A. Sommerfeld, Atombau and Spektrallinien, II. Band, 2nd ed. (Vieweg, Braunschweig, 1939), p. 216.

10) For a discussion of this point and references to the original work on the Klein-Gordon equation we refer to H. Kragh, Am. J. Phys. 52 (1984) 1024. 